Archives de sciences sociales des religions

116 | octobre - décembre 2001

Varia

\title{
Anne-Hélène Trottier, Fakir. La quête d'un Bâul musulman
}

Paris, L'Harmattan, 2000, 223 p. (bibliogr., glossaire, illustr.)

André Padoux

\section{OpenEdition}

\section{Journals}

Édition électronique

URL : http://journals.openedition.org/assr/1563

DOI : 10.4000/assr. 1563

ISSN : $1777-5825$

\section{Éditeur}

Éditions de l'EHESS

Édition imprimée

Date de publication : 2 octobre 2001

Pagination : 93-156

ISBN : 2-222-96712-0

ISSN : 0335-5985

\section{Référence électronique}

André Padoux, "Anne-Hélène Trottier, Fakir. La quête d'un Bâul musulman », Archives de sciences sociales des religions [En ligne], 116 | octobre - décembre 2001, document 116.38, mis en ligne le 14 novembre 2005, consulté le 24 septembre 2020. URL : http://journals.openedition.org/assr/1563 ; DOI : https://doi.org/10.4000/assr.1563 
'impensée' parce que la modernité, en rupture avec le religieux, n'a pas su saisir la dimension historico-politique de l'existence juive. Ce qui explique, selon lui, que la 'question juive' soit au cœur même de la démocratie, et en même temps que ni Sartre, ni Arendt, malgré l'acuité de leurs analyses, n'aient pu donner à cette question une réponse satisfaisante. D'où, dans une seconde partie : "L'Homme-du-Citoyen », un effort pour mettre à jour la faille de la réflexion démocratique elle-même. Le projet des révolutionnaires de 1789 de "régénérer 》 le genre humain, reposant sur l'ambiguïté de la notion de 'nature', à la fois abstraite (la 'nature humaine' de l'ordre de la rationalité formelle qui fonde les Droits de l'homme) et infra-rationnelle (la 'nature' empirique, biologique), entraîne le rejet de l'hétéronomie et la forclusion de l'origine. C'est-à-dire, finalement, conjointement la négation de l'identité juive et la raciation de l'existence juive, le déplacement de la 'différence' là où elle n'aurait pas dû se trouver. Mais plus globalement, c'est à «l'indétermination démocratique »-S.T. reprend ici une expression de Claude Lefort, mais dans un sens un peu différent puisqu'il la définit comme «l'absence de l'Homme dans l'institution de l'Homme » qu'il rattache le disfonctionnement de la modernité. La troisième partie : «Le Juif-del'Homme », tente alors de répondre à la question posée, la réalité de la Shoah dans une Europe qui a inventé l'idée que tous les hommes sont égaux. À travers l'examen de "l'énigme de la singularité juive» et de «l'énigme (de la notion de) 'peuple'», c'est «à un nouvel âge de la démocratie » (il y met un point d'interrogation) qu'il nous invite. Une démocratie qui ne limite plus sa référence au modèle athénien de l'enracinement ou de l'autochtonie, tel qu'il a été construit comme un idéal par le $\mathrm{XIX}^{\mathrm{e}}$ siècle, mais qui s'inspire aussi du modèle biblique - précisément écarté par ce même XIX ${ }^{\mathrm{e}}$ siècle - de la fondation d'une collectivité dans l'exil et par la loi, telle qu'elle s'est élaborée à travers la sortie d'Égypte et la traversée du désert. Une démocratie qui, ce faisant, s'affranchit de la tentation de la totalité et donc du 'pouvoir', et atteint l'universel dans l'alliance de l'identité et de l'hospitalité...

L'ouvrage que nous propose ici l'A., qui enseigne la sociologie de la politique et la sociologie de la religion à l'Université de Nanterre, et est aussi Directeur du Collège des études juives de l'Alliance israélite universelle, est souvent suggestif. Par-delà le problème de la Shoah et de sa signification, il nous invite à réfléchir aux fondements mêmes de la 'démocratie' et à ce qui la rendrait capable d'articuler l'égalité des citoyens et la différence de chaque individu.

Yves Chevalier.

116.38

TROTTIER (Anne-Hélène).

Fakir. La quête d'un Bâul musulman. Paris, L'Harmattan, 2000, 223 p. (bibliogr., glossaire, illustr.).

Si les Bâuls, ces poètes-chanteurs mystiques hindous du Bengale, sont devenus célèbres (et sont relativement bien étudiés) depuis le temps où Rabindranath Tagore les avait "découverts » et exaltés, et surtout depuis qu'ils chantent à la radio et apparaissent dans les concerts de "musiques du monde », les Fakirs, qui sont des Bâuls musulmans, sont restés presque inconnus. Cela explique l'intérêt que leur a porté l'anthropologue A.-H.T., spécialiste du Bengale (et musicienne), désireuse d'étudier un groupe moins important, limité dans son implantation géographique à quelques districts de l'est du Bengale indien et à un petit coin du Bangladesh, groupe à bien des égards mystérieux et dont on pouvait se demander comment il pouvait vivre une quête à forte composante sexuelle, ainsi que des pratiques et des notions marquées de tantrisme, en tant que musulmans et en milieu villageois islamique. L'auteur a vécu auprès de ces Fakirs au cours de plusieurs années, les suivant dans les foires religieuses où il leur arrive de chanter et vivant dans l'intimité de l'un d'eux, dans sa famille et son lignage spirituel, se coulant en quelque sorte « dans le mode de fonctionnement traditionnel de la transmission de maître à disciple » (p. 16), de façon à arriver, dans la mesure du possible, à une compréhension globale et approfondie, " participante » presque, de son sujet. D'où un ouvrage attachant, pénétrant et surtout riche d'informations tant sur ce groupe que sur certains aspects importants de la réalité socioreligieuse indienne.

Comme les Bâuls, les Fakirs suivent une voie d'expérience directe, ce qu'exprime leur commune désignation de bartamàn panthi. Ils diffèrent toutefois des Bâuls en ce qu'ils suivent toujours une pratique ésotérique initiatique à base corporelle. Tout en s'affirmant fidèles d'Allah, ils refusent les normes islamiques orthodoxes, leur religion étant centrée sur l'homme - c'est un manush dharma -, et plus spécialement sur le corps (indissociable de l'esprit). Les Fakirs chantent en s'accompagnant des mêmes instruments traditionnels que les Bâuls. Mais c'est un chant individuel ou à 
deux, qui n'est pas destiné à séduire une audience, mais plutôt à l'échange entre initiés, au plan où la parole chantée s'efforce d'exprimer ou de transmettre ce que le langage parlé ne peut pas dire - un peu comme un mantra. Le chant serait ainsi à la fois inspiration et incarnation de la quête. Il sert à dire et à rappeler les enseignements ésotériques de la secte, tout en ayant un rôle corporel puisqu'il est produit par le souffle, dont le contrôle est un aspect important de la pratique fakir. L'A. tente d'exprimer ce rôle du chant en une formule un peu complexe quand elle écrit (p. 93) qu'il est peut-être «la pointe émergée d'un iceberg fait de techniques ésotériques du corps (respiratoires, musicales, sexuelles), qui partagent des fils conducteurs communs, les sens et les souffles, et dont la mobilisation vers un objectif commun produit le sens autre que les chants lui réinjectent en boucle. »

A.-H.T. consacre la plus grande partie du livre (3 chapitres) à la sâdhanâ du Fakir, c'est-à-dire à sa quête du divin en tant que présent en ce monde, quête où se mêlent inextricablement les doctrines ésotériques et le concret des pratiques corporelles, sans oublier les contraintes de l'existence dans la pauvreté d'un village bengali. Les Fakirs sont en effet (nécessairement) mariés, ont une famille et vivent d'un travail manuel, généralement agricole. Musulmans, ils se disent de tradition soufie. Sans former une confrérie, ils se rattachent à une tradition ésotérique dont le maître principal - et l'auteur de nombre de leurs chants - fut le Fakir Lalan, qui aurait vécu de 1784 jusqu'à la fin du $\mathrm{XIX}^{\mathrm{e}}$ siècle et qui appartenait lui-même à une lignée issue de Mohiuddin Chishti (XII ${ }^{\mathrm{e}}-\mathrm{XIII}{ }^{\mathrm{e}}$ siècles). Une grande importance est attachée à la lignée ésotérique de transmission de la sagesse, tout apprenti Fakir devant avoir un maître (guru, murshid) de la tradition dans laquelle il sera initié. Mais si la tradition fakir est soufie, elle l'est de façon bien particulière, car elle intègre dans son enseignement comme dans ses pratiques des éléments de religion populaire bengalie, des traits hindous et bouddhiques de caractère tantrique. Secret, cet enseignement ne peut être évoqué qu'entre initiés. Il est transmis oralement par la parole du guru et par les chants, où il s'exprime en un langage conventionnel, le sandhabhâsha, la langue 'intentionnelle' ou 'crépusculaire' (désignation propre en réalité au bouddhisme tantrique), qui en dissimule le sens au profane.

Notons au passage que la musique - le chant et les instruments qui l'accompagnent - peut aider à transmettre une vérité que la parole ordinaire ne peut exprimer. Le chant lui-même, d'ailleurs, porté par le souffle, apparaît ici comme une des «techniques du corps» qui, comme le notait Marcel Mauss (à qui l'A. se réfère), sont souvent associées aux états mystiques.

Quant aux doctrines, la religion des Fakirs se dit religion de l'Homme, manush dharma. Elle affirme la présence du divin en l'être humain sans référence à un dieu transcendant, notion islamique fondamentale pourtant, mais qui paraît inutile aux Fakirs, qui rejettent les pratiques rituelles et observances de l'islam comme sans objet. "Le divin ne réside pas dans ces lieux, mais dans le temple par excellence qu'est pour le Fakir, son propre corps », ce corps qui - selon la conception généralement indienne/hindoue, mais plus spécialement tantrique - est un microcosme correspondant en tout point au macrocosme. "C'est toi le Coran », dit le Fakir Mansur. La doctrine et les pratiques ne cessent de faire état et de reposer sur ces correspondances entre le corps et le monde (le milieu géographique bengali où vivent les Fakirs). Mais, si le corps humain est analogiquement identique au cosmos, l'idéologie de la voie fakir s'inscrit à contre-courant du mouvement cosmique par une volonté d'utiliser le corps en le transcendant. Dans cette voie " corporéocentrée » (dehakendrika), dans cette doctrine ou science du corps (dehabadi, dehatattva), la représentation du corps est très largement celle qui se trouve dans les systèmes tantriques, avec ses canaux où circulent les " souffles », ses centres d'énergie, les chakra, ainsi qu'avec l'énergie fondamentale divino-humaine de la kundalinî, puissances qu'éveillent et maîtrisent des pratiques particulières notamment d'ordre sexuel. Celles-ci reposent spécialement sur la force qu'est censée posséder la femme. Elles visent aussi, essentiellement, à préserver et transformer la semence masculine, le $b \hat{\imath} j$. Les Fakirs pratiquent ainsi, avec leur femme, selon des méthodes traditionnellement enseignées, un coïtus reservatus où le sperme n'est pas éjaculé, mais, mêlé aux sécrétions féminines et spécialement au sang menstruel (l'union au moment des règles étant recommandée chez les Fakirs comme chez les Bâuls) est conservé et transformé en assurant dès lors au Fakir la perfection inséparablement spirituelle et corporelle. L'idéal est de réaliser l'état de jyante mara, de mort dans la vie: une vie qui n'est plus soumise à tout ce qui normalement la menace.

L'idée de la divinité du corps fait aussi que tout ce qu'il produit est considéré comme chargé de puissance. D'où la consommation rituelle par le Fakir et sa compagne des « quatre 
lunes» que sont les selles, l'urine et autres sécrétions sexuelles, le sang menstruel et le sperme - pratique dont on trouve déjà des formes dans des tantras datant des premiers siècles de notre ère. L'A. a pu recueillir des indications assez précises sur les règles de conduite liées à ces pratiques, qu'elles concernent l'alimentation (végétarienne), le contrôle des souffles (prânâyâma - vieille pratique yogique) et même la pratique sexuelle (yugalsâdhana) qui suppose tout un apprentissage de la part du Fakir comme de son épouse puisqu'il s'agit, par leur action conjuguée, de connaître un plaisir prolongé, que n'interrompt pas l'orgasme et qui, de plus, mène à l'assimilation corporelle transformante de l'énergie divine ainsi éveillée.

L'A fait remarquer en terminant que si le rôle de la femme, seule à posséder naturellement la puissance, est ainsi exalté, celle-ci n'en est pas pour autant traitée avec un respect particulier. Sans doute l'épouse fakir a-t-elle sur les autres femmes de son village l'avantage de n'avoir qu'un (ou deux) enfants - il faut en effet un fils pour continuer la lignée spirituelle. Mais, pour le reste, sa condition ne diffère guère de celle de ses consœurs. Là, comme ailleurs dans la tradition indienne/hindoue, si la femme est exaltée, elle l'est par les hommes et à leur bénéfice. Si elle est la source, c'est celle où les hommes vont boire.

Le cas des Fakirs, on le voit, outre son intérêt propre, apparaît comme posant, lui aussi, la question de la vision indienne de l'être humain : celle de sa place dans le cosmos, celle des homologies micro-macrocosmiques, celle de l'utilisation du corps (et de ses substances constitutives ou sécrétions) à des fins qui à la fois le transforment et le transcendent. Il n'est pas indifférent de voir un petit groupe musulman contemporain mettre en pratique des notions pouvant paraître propres au tantrisme et dont les racines plongent au plus profond du sol de l'Inde.

Tant par ses descriptions précises et son intelligence des situations que par les nombreux problèmes connexes qu'il soulève ou évoque à l'esprit du lecteur, ce petit livre me semble être d'un grand intérêt. Au-delà, en effet, des Fakirs, qui, déjà, méritent grandement notre attention, c'est un aspect fondamental de la vision indienne de l'être humain et de sa présence au monde qui est abordé ici.

André Padoux.
Islam in the African-American Experience. Bloomington-Indianapolis, Indiana University Press, 1997, (bibliogr., index, illustr.).

Islam in the African-American Experience décrit le rôle que joue l'islam dans la formation de l'identité des Noirs américains au $\mathrm{XX}^{\mathrm{e}}$ siècle. L'auteur remonte à la traite des esclaves, revenant ainsi à l'histoire d'un " islam global » entre Moyen-Orient, Afrique de l'Ouest et Amérique du Nord avant la guerre civile. Il s'appuie notamment sur des récits d'esclaves noirs et musulmans et des rapports de missionnaires chrétiens pour montrer que les idéologies qui émergent au $\mathrm{XX}^{\mathrm{e}}$ siècle par le biais d'Elijah Muhammad par exemple, ne sont pas radicalement nouvelles. Il dessine donc une continuité entre d'une part l'islam ouest-africain, vécu puis découvert ou re-découvert par les Noirs d'Amérique et l'idée d'un nationalisme noir. L'islam aurait ainsi fourni une identité nouvelle et une généalogie à une population qui en avait été brutalement privée, d'où l'insistance de l'A. sur la notion de "naming and renaming» (p. 4), et d'un " jihâd des mots » comme processus de recréation d'une identité. L'idée d'un séparatisme racial remonte pour l'A. non pas au nationalisme noir américain, mais à une norme déjà présente en Afrique avant la traite des esclaves vers l'Amérique. Autre originalité de l'ouvrage: l'importance, selon l'A., de l'influence du mouvement d'origine indienne des Ahmadiyya dans l'évolution de l'islam noir américain, notamment sur l'idéologie de Elijah Muhammad, en introduisant une tension entre nationalisme exclusivement noir, et islam multi-racial.

L'ouvrage se compose de deux parties inégales : les racines de l'islam noir (36 p.), puis ses prophètes (172 p.).

Dans la première partie, l'A. revient aux récits de vie des premiers esclaves musulmans en Amérique du Nord, qui restent malheureusement très partiels, et nous informent finalement assez peu sur leur quotidien, et surtout sur la pratique de leur religion. Il semblerait que les esclaves musulmans aient été relativement plus instruits, et aient ainsi produit, par le biais de leur religion, de quoi alimenter la notion de « résistance» à l'esclavage. Mais la démonstration n'est pas convaincante, car les documents sur lesquels l'A. se fonde sont peu nombreux et parce qu'il faudrait pour étayer une telle hypothèse comparer au plus près avec ce que firent les esclaves noirs non musulmans, qui ne sont pas évoqués dans cette première partie. L'A. généralise à partir de l'exemple de quelques esclaves musulmans lettrés: "For African 